\title{
Fate of transgenic DNA and evaluation of metabolic effects in goats fed genetically modified soybean and in their offsprings - RETRACTION
}

\author{
R. Tudisco, V. Mastellone, M. I. Cutrignelli, P. Lombardi, F. Bovera, N. Mirabella, G. Piccolo, \\ S. Calabrò, L. Avallone and F. Infascelli
}

doi:10.1017/S1751731110000728, first published online by Cambridge University Press, 5 May 2010.

The manuscript 'Fate of transgenic DNA and evaluation of metabolic effects in goats fed genetically modified soybean and in their offsprings' by Tudisco R, Mastellone V, Cutrignelli Ml, Lombardi P, Bovera F, Mirabella N, Piccolo G, Calabro S, Avallone L, Infascelli $F$ was submitted to animal on 16 March 2009. After peer-review and revision, it was accepted for publication on 29 March 2010 and published in 2010.

From late September 2015, we received several expressions of concern from third parties that the electrophoresis gels presented might have been subject to unwarranted digital manipulations (added and hidden bands or zones, including in the control samples and the DNA ladder). A detailed independent investigation was carried out by animal in accordance with the Committee on Publication Ethics (COPE) guidelines. This investigation included an analysis of the claims using the figures as submitted, and reassessment of the article by one of the original peer-reviewers in light of the results of the analysis. The authors were notified of our concerns and asked to account for the observed irregularities. In the absence of a satisfactory explanation, the institution was asked to investigate further. The University of Naples concluded that multiple heterogeneities were likely attributable to digital manipulation, raising serious doubts on the reliability of the findings.

Based on the results of all investigations, we have decided to retract the article.

\section{Reference}

Tudisco $\mathrm{R}$, Mastellone V, Cutrignelli MI, Lombardi P, Bovera F, Mirabella N, Piccolo G, Calabrò S, Avallone $L$ and Infascelli $F$ 2010. Fate of transgenic DNA and evaluation of metabolic effects in goats fed genetically modified soybean and in their offsprings. Animal 4, 1662-1671. 